\title{
DATA PAGE
}

\section{Q20 - biotech stocks nosedive}

With the onset of COVID-19, biotech stocks, along with all the other major stock market indices, took a nose dive, from mid-March. The biotech index performed a little better than the other indices by the end of the quarter. In the weeks before the pandemic announcement, money still flowed into the sector from both the public and private markets. As yet, evidence of an economic downturn is not displayed in the numbers.

\section{Stock market performance}

Biotech stocks react to the economic downturn.

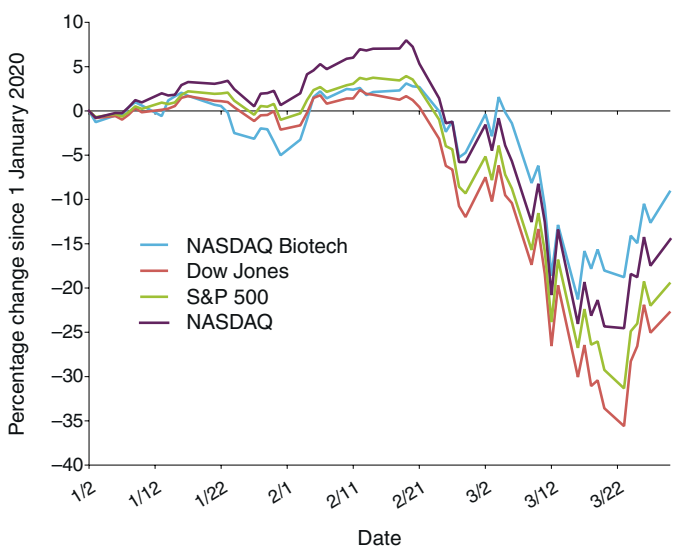

Global biotech intial public offerings

The US had a bumper quarter in terms of IPO funding.

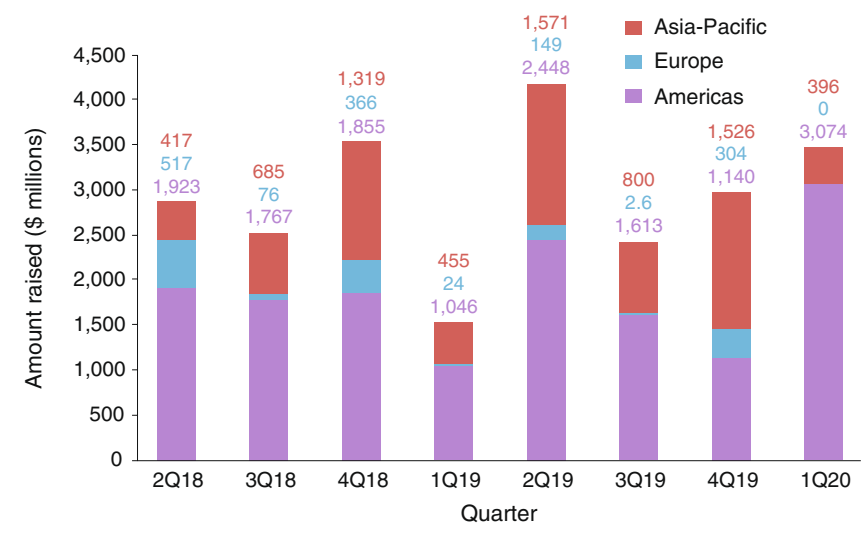

\section{Number of IPOs}

\begin{tabular}{lllllllll} 
& 2Q18 & 3Q18 & 4Q18 & 1Q19 & 2Q19 & 3Q19 & 4Q19 & 1Q20 \\
\hline Asia-Pacific & 4 & 7 & 13 & 3 & 8 & 6 & 17 & 2 \\
Europe & 6 & 1 & 5 & 2 & 2 & 1 & 3 & 0 \\
Americas & 17 & 19 & 11 & 18 & 18 & 9 & 13 & 10 \\
\hline
\end{tabular}

Source: BCIQ BioCentury Online Intelligence.
Global biotech financing

Debt continued to be an important funding source.

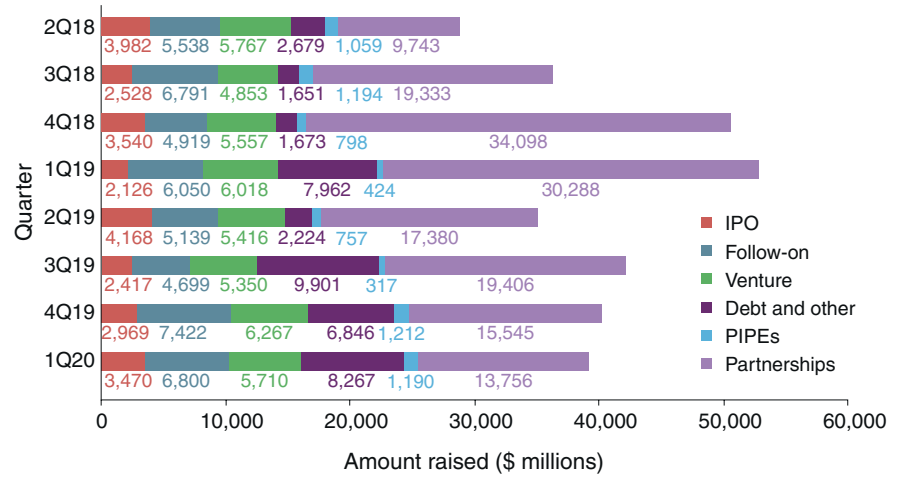

PIPE, private investment in public equity. Source: $\mathrm{BCIQ}$ BioCentury Online Intelligence.

\section{Global biotech venture capital investment}

In the United States and Europe, taken together, risk capital was more plentiful than in any 2019 quarter.

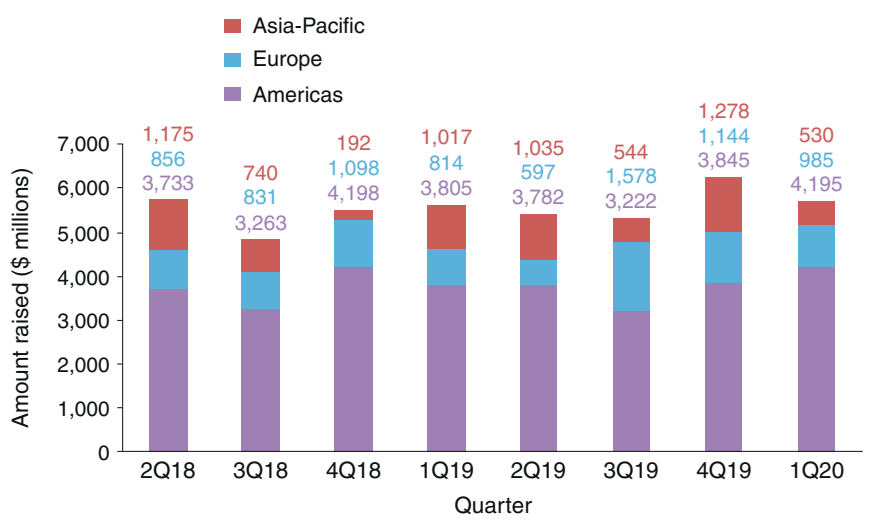

\section{Number of rounds}

\begin{tabular}{lllllllll} 
& $\mathbf{2 Q 1 8}$ & $\mathbf{3 Q 1 8}$ & $\mathbf{4 Q 1 8}$ & $\mathbf{1 Q 1 9}$ & $\mathbf{2 Q 1 9}$ & $\mathbf{3 Q 1 9}$ & $\mathbf{4 Q 1 9}$ & $\mathbf{1 Q 2 0}$ \\
\hline Asia-Pacific & 23 & 15 & 7 & 18 & 18 & 15 & 27 & 12 \\
Europe & 28 & 29 & 35 & 29 & 39 & 41 & 43 & 49 \\
Americas & $\mathbf{9 6}$ & 82 & 85 & 60 & 104 & 86 & 104 & 99 \\
\hline
\end{tabular}

Source: BCIQ BioCentury Online Intelligence. 
IPOs

\begin{tabular}{llll}
\hline Company (principal underwriters) & $\begin{array}{l}\text { Amount } \\
\text { raised } \\
\text { (\$ millions) }\end{array}$ & $\begin{array}{l}\text { Date } \\
\text { completed }\end{array}$ & $\begin{array}{l}\text { Change in } \\
\text { stock price } \\
\text { (as of } \\
\mathbf{4}\end{array}$ \\
\hline Bio-Thera Solutions
\end{tabular}

Source: BCIQ BioCentury Online Intelligence.

\section{Mergers and acquisitions}

\begin{tabular}{llll} 
Target & Acquirer & $\begin{array}{l}\text { Value } \\
\text { (\$ millions) }\end{array}$ & $\begin{array}{l}\text { Date } \\
\text { announced }\end{array}$ \\
\hline Dermira & Eli Lilly & 1,000 & 10 January \\
\hline Ritter Pharmaceuticals & Qualigen & Reverse merger & 21 January \\
\hline Neon Therapeutics & BioNTech & 67 & 16 January \\
\hline PharmAkea Therapeutics & Galecto Biotech & Merger & 7 January \\
\hline Onspira Therapeutics & Altavant & Undisclosed & 8 January \\
\hline Attenua & Coda Biotherapeutics & Undisclosed & 5 February \\
\hline
\end{tabular}

Source: $\mathrm{BCIQ}$ BioCentury Online Intelligence.

\section{Licensing/collaboration}

\begin{tabular}{|c|c|c|c|}
\hline Researcher & Partner & $\begin{array}{l}\text { Up-front } \\
\text { cash } \\
\text { (\$ millions) }\end{array}$ & Description \\
\hline $\begin{array}{l}\text { Sangamo } \\
\text { Therapeutics }\end{array}$ & Biogen & 125 & $\begin{array}{l}\text { Sangamo grants Biogen exclusive } \\
\text { worldwide rights to develop and } \\
\text { commercialize gene regulation therapies } \\
\text { to treat neurological diseases }\end{array}$ \\
\hline Novartis & $\begin{array}{l}\text { Otsuka } \\
\text { Pharmaceutical }\end{array}$ & 90 & $\begin{array}{l}\text { Otsuka enters into co-promotion } \\
\text { agreement in Japan for small-molecule } \\
\text { cocktail of the neprilysin inhibitor } \\
\text { sacubitril plus angiotensin receptor } \\
\text { inhibitor valsartan }\end{array}$ \\
\hline $\begin{array}{l}\text { Dermavant } \\
\text { Sciences }\end{array}$ & Japan Tobacco & 60 & $\begin{array}{l}\text { Dermavant enters into exclusive license } \\
\text { agreement with Japan Tobacco for } \\
\text { development and commercialization of } \\
\text { tapinarof, a first-in-class small-molecule } \\
\text { aryl hydrocarbon receptor agonist, in Japan }\end{array}$ \\
\hline $\begin{array}{l}\text { Nurix } \\
\text { Therapeutics }\end{array}$ & Sanofi & 55 & $\begin{array}{l}\text { Nurix partners with Sanofi to develop and } \\
\text { commercialize protein degradation drugs } \\
\text { to treat multiple undisclosed indications }\end{array}$ \\
\hline AstraZeneca & $\begin{array}{l}\text { RedHill } \\
\text { Biopharma }\end{array}$ & 53 & $\begin{array}{l}\text { AstraZeneca grants RedHill rights to } \\
\text { commercialize Movantik (naloxegol) } \\
\text { to treat opioid-induced constipation } \\
\text { worldwide, excluding Europe, Canada } \\
\text { and Israel }\end{array}$ \\
\hline $\begin{array}{l}\text { Adaptimmune } \\
\text { Therapeutics }\end{array}$ & $\begin{array}{l}\text { Astellas } \\
\text { Pharma }\end{array}$ & 50 & $\begin{array}{l}\text { Astellas partners with Adaptimmune } \\
\text { to co-develop and co-commercialize } \\
\text { stem-cell-derived allogeneic T cell } \\
\text { therapies to treat cancer }\end{array}$ \\
\hline $\begin{array}{l}\text { Kyverna } \\
\text { Therapeutics }\end{array}$ & $\begin{array}{l}\text { Gilead } \\
\text { Sciences }\end{array}$ & 17.5 & $\begin{array}{l}\text { Kyverna partners with Gilead to develop } \\
\text { engineered T cell therapies for the } \\
\text { treatment of autoimmune disease based } \\
\text { on Kyverna's synthetic } T_{\text {reg }} \text { platform and } \\
\text { synNotch technology from Kite }\end{array}$ \\
\hline $\begin{array}{l}\text { eFFECTOR } \\
\text { Therapeutics }\end{array}$ & Pfizer & 15 & $\begin{array}{l}\text { eFFECTOR Therapeutics grants Pfizer } \\
\text { worldwide, exclusive rights to develop } \\
\text { and commercialize small-molecule } \\
\text { inhibitors of eukaryotic initiation factor } \\
4 E \text { to treat cancer }\end{array}$ \\
\hline $\begin{array}{l}\text { Camp4 } \\
\text { Therapeutics }\end{array}$ & Biogen & 15 & $\begin{array}{l}\text { Biogen partners with Camp4 to discover } \\
\text { targets to address neurodegenerative and } \\
\text { neurological diseases }\end{array}$ \\
\hline GlycoMimetics & Apollomics & 9 & $\begin{array}{l}\text { GlycoMimetics grants Apollomics } \\
\text { exclusive rights to its E-selectin antagonist, } \\
\text { GMI-1687, to treat acute myeloid leukemia }\end{array}$ \\
\hline Evotec & Bayer & 7.2 & $\begin{array}{l}\text { Evotec partners with Bayer to develop and } \\
\text { commercialize drug candidates to treat } \\
\text { polycystic ovary syndrome }\end{array}$ \\
\hline
\end{tabular}

Source: BCIQ BioCentury Online Intelligence.

\section{Laura DeFrancesco}

Senior Editor, Nature Biotechnology.

Published online: 11 May 2020

https://doi.org/10.1038/s41587-020-0516-1 